\title{
VIETORIS-BEGLE THEOREM AND SPECTRA
}

\author{
JERZY DYDAK AND GEORGE KOZLOWSKI
}

(Communicated by James E. West)

\begin{abstract}
The following generalization of the Vietoris-Begle Theorem is proved: Suppose $\left\{E_{k}\right\}_{k \geq 1}$ is a CW spectrum and $f: X^{\prime} \rightarrow X$ is a closed surjective map of paracompact Hausdorff spaces such that Ind $X=m<\infty$. If $f^{*}: E^{k}(x) \rightarrow E^{k}\left(f^{-1}(x)\right)$ is an isomorphism for all $x \in X$ and $k=m_{0}$, $m_{0}+1, \ldots, m_{0}+m$, then $f^{*}: E^{n}(X) \rightarrow E^{n}\left(X^{\prime}\right)$ is an isomorphism and $f^{*}: E^{n+1}(X) \rightarrow E^{n+1}\left(X^{\prime}\right)$ is a monomorphism for $n=m_{0}+m$.
\end{abstract}

Given a CW spectrum $E=\left\{E_{k}\right\}_{k \geq 1}$ and a pointed CW complex $K$, one has cohomology groups $E^{n}(K)$ for each integer $n$ (see [Sw, Chapter 8]). They are defined as homotopy classes from the suspension spectrum of $K$ to $\Sigma^{n} E$, where $\Sigma^{n} E$ is defined by $\Sigma^{n} E_{k}=E_{k+n}$. In the case of an $\Omega$-spectrum (i.e., where the natural map $E_{k} \rightarrow \Omega E_{k+1}$ is a homotopy equivalence for all $\left.k\right), E^{n}(K)$ is isomorphic to $\left[K, E_{n}\right]$ (see [Sw, Theorem 8.42]). The groups $E^{n}(X), X$ being any pointed topological space, are defined as $\operatorname{dir} \lim \left\{E^{n}\left(X_{\alpha}\right), p_{\alpha \beta}^{*}, \Lambda\right\}$, where $\left\{X_{\alpha}, p_{\alpha \beta}, \Lambda\right\}$ is the Čech system of $X$ (see [D-S, p. 21] for the definition of the Čech system of $X$ ). In this way one gets the Čech extension of the functor $E^{n}$ from pointed CW complexes to pointed spaces (see [D] for a general discussion of Čech extensions of functors). Again, if $\left\{E_{k}\right\}_{k \geq 1}$ is an $\Omega$-spectrum, then $E^{n}(X)$ is isomorphic to $\left[X, E_{n}\right]$. A basic result is that every spectrum $\left\{E_{k}\right\}_{k \geq 1}$ is isomorphic to an $\Omega$-spectrum (see [B, part 10 of Chapter II]). Essentially, the $n$th term of that spectrum is the telescope of $E_{n} \rightarrow \Omega E_{n+1} \rightarrow \Omega^{n} E_{n+2} \rightarrow \cdots$.

In the case of an unpointed topological space $X$, we define the unreduced cohomology $E^{n}(X)$ as $E^{n}\left(X^{+}\right)$, where $X^{+}$is $X$ with a discrete base point added.

Recall the classical Vietoris-Begle Theorem (see [S, p. 344]):

Vietoris-Begle Theorem. Let $f: X^{\prime} \rightarrow X$ be a closed surjective map of paracompact Hausdorff spaces. Assume that there is an $n \geq 0$ such that $\widetilde{H}^{k}\left(f^{-1}(x)\right)=0$ (reduced Čech cohomology) for all $x \in X$ and for $k<n$. Then $f^{*}: H^{k}(X) \rightarrow$ $H^{k}\left(X^{\prime}\right)$ is an isomorphism for $k<n$ and a monomorphism for $k=n$.

Received by the editors February 18, 1990 and, in revised form, March 29, 1990.

1980 Mathematics Subject Classification (1985 Revision). Primary 55N05; Secondary 55N20, $55 \mathrm{P} 20$.

Key words and phrases. Vietoris-Begle theorem, spectra, cohomology. 
A natural question arises: Since Čech cohomology corresponds to the Eilenberg-MacLane spectrum $\{K(G, k)\}_{k \geq 1}$, is there a generalization of the above theorem to arbitrary spectra?

The naive approach of replacing $\widetilde{H}^{k}\left(f^{-1}(x)\right)=0$ by $E^{k}\left(f^{-1}(x)\right) \approx E^{k}(\{x\})$ does not work. An example of that is Taylor's cell-like map (see [T]) $f: X \rightarrow Q$ onto the Hilbert cube $Q$ such that $\widetilde{K}(X)$ is nonzero $(\widetilde{K}$ is the reduced complex $K$ theory which is the cohomology theory of the spectrum BU, the periodic spectrum $U, B U, \ldots)$. This example was later modified by J. Keesling [K] who produced a cell-like map $g: Q \rightarrow Y$ with $\widetilde{K}(Y) \neq 0$.

The aim of this paper is to prove suitable generalizations of the Vietoris-Begle Theorem involving arbitrary unreduced spectral cohomology. In this task we were guided by Kozlowski's result (see [Ko] or [D-S]) proved in 1975.

Theorem (G. Kozlowski). For closed maps $f: X \rightarrow Y$ of metrizable spaces such that $\left[f^{-1}(A), K\right]=[A, K]$ for any $\mathrm{CW}$ complex $K$ and any closed subset $A$ of $Y$, the image $Y$ is an ANR provided $X$ as an ANR.

Kozlowski's proof involved a trick: he showed that certain maps from $X$ to $K$ can be extended over the mapping cylinder $M(f)$ of $f$. Then he replaced $X$ by the double mapping cylinder $\operatorname{DM}(f)$ of $f$ (the union of two copies of $M(f)$ sewn along $X$ ) and used the previous construction to relate any two different extensions. The meaning of this trick is that it echoes the Mayer-Vietoris exact sequence (once you prove that a certain homomorphism is onto you get that the next one is trivial, for free). In this paper we formalize this observation: the trick becomes Lemma 3 (the mapping cone $C_{p}$ of $p: \operatorname{DM}(f) \rightarrow Y$ is homeomorphic to the reduced suspension $S\left(C_{f}\right)$ of the mapping cone of $f$ ) and the whole approach resembles a Puppe exact sequence. Notice that Theorem $B$ implies the results of [D-K] (the goal of that paper was to unify Vietoris-Begle Theorem and cell-like maps on spaces of finite deformation dimension).

Theorem A. Suppose $\left\{E_{k}\right\}_{k \geq 1}$ is a CW spectrum and $f: X^{\prime} \rightarrow X$ is a closed surjective map of paracompact Hausdorff spaces such that Ind $X=m<\infty$. If $f^{*}: E^{n}(x) \rightarrow E^{n}\left(f^{-1}(x)\right)$ is an isomorphism for all $x \in X$ and $n=$ $m_{0}, m_{0}+1, \ldots, m_{0}+m$, then $f^{*}: E^{n}(X) \rightarrow E^{n}\left(X^{\prime}\right)$ is an isomorphism and $f^{*}: E^{n+1}(X) \rightarrow E^{n+1}\left(X^{\prime}\right)$ is a monomorphism for $n=m_{0}+m$.

Remark. Ind $X$ is the large inductive dimension of $X$ : Ind $\varnothing=-1$ and Ind $X$ $\leq m$ means that for any neighborhood $U$ of a closed subset $A$ of $X$, there is a neighborhood $V$ of $A$ in $U$ with $\operatorname{Ind}(\operatorname{cl}(V)-V) \leq m-1$.

Theorem B. Suppose $\left\{E_{k}\right\}_{k \geq 1}$ is a CW spectrum and $f: X^{\prime} \rightarrow X$ is a closed surjective map of paracompact Hausdorff spaces such that the following conditions are satisfied:

(a) $f^{*}: E^{n-1}(A) \rightarrow E^{n-1}\left(f^{-1}(A)\right)$ is an epimorphism for all closed subsets $A$ of $X$, and 
(b) $f^{*}: E^{n}(A) \rightarrow E^{n}\left(f^{-1}(A)\right)$ is a monomorphism for all closed subsets $A$ of $X$.

If $f^{*}: E^{n}(x) \rightarrow E^{n}\left(f^{-1}(x)\right)$ is an isomorphism for all $x \in X$, then $f^{*}$ : $E^{n}(X) \rightarrow E^{n}\left(X^{\prime}\right)$ is an isomorphism and $f^{*}: E^{n+1}(X) \rightarrow E^{n+1}\left(X^{\prime}\right)$ is a monomorphism.

Remark. Obviously, conditions (a) and (b) are derived from Kozlowski's Theorem.

The proofs of Theorems A and B will depend on Lemmas 1-4 below.

Given a map $f: X^{\prime} \rightarrow X$ and a subset $A$ of $X, f^{-1}(A)$ is denoted by $A^{\prime}$ and the map $A^{\prime} \rightarrow A$ defined by $f$ is denoted by $f_{A}$.

Let $C_{f}=M(f) / X^{\prime}$ be the mapping cone of a map $f: X^{\prime} \rightarrow X . q_{f}: M(f) \rightarrow$ $C_{f}$ denotes the quotient map from the mapping cylinder $M(f)$ of $f$ to $C_{f}$. Given a map $f: X^{\prime} \rightarrow X$ and a space $E, f^{*}:[X, E] \rightarrow\left[X^{\prime}, E\right]$ is called monic provided that for any map $g: X \rightarrow E, g f \approx$ const implies $g \approx$ const.

Lemma 1. Suppose $f: X^{\prime} \rightarrow X$ is a map such that $f^{*}:[X, \Omega E] \rightarrow\left[X^{\prime}, \Omega E\right]$ is onto. Then $q_{f}^{*}:\left[C_{f}, E\right] \rightarrow[M(f), E]$ is monic.

Proof. Suppose $g: C_{f} \rightarrow E$ is a map such that $g \mid X \approx$ const. We may assume $g \mid X=$ const (by homotoping $g$ ). Then $g$ factors as $C_{f} \rightarrow \Sigma X^{\prime} \rightarrow E$, which in turn factors (up to homotopy) as $C_{f} \rightarrow \Sigma X^{\prime} \rightarrow \Sigma X \rightarrow E$. Notice that $C_{f} \rightarrow \Sigma X$ is null-homotopic, as it factors as $C_{f} \rightarrow C(X) \rightarrow \Sigma X, C(X)$ being the cone over $X$. Thus $g \approx$ const .

Lemma 2. Suppose $f: X^{\prime} \rightarrow X$ is a map such that $q_{f}^{*}:\left[C_{f}, E\right] \rightarrow[M(f), E]$ is monic. If $g, h: M(f) \rightarrow E$ are two null-homotopic maps such that $g \mid X^{\prime}=$ $h \mid X^{\prime}$, then $g \approx h$ rel. $X^{\prime}$.

Proof. We need to extend the map $G: M(f) \times\{0,1\} \cup X^{\prime} \times I \rightarrow E$, where $G|M(f) \times\{0\}=g, G| M(f) \times\{1\}=h$, and $G(x, t)=g(x)$ for $(x, t) \in X^{\prime} \times I$, over $M(f) \times I$. Since $G \mid X \times\{0\} \approx$ const, $G$ extends to $G^{\prime}:(M(f) \times\{0,1\} \cup$ $\left.X^{\prime} \times I\right) \cup C(X \times\{0\}) \rightarrow E$, where $C(X \times\{0\})$ is the cone over $X \times\{0\}$. Notice that $\left.M(f) \times\{0,1\} \cup X^{\prime} \times I\right) \cup C\left(X \times\{0\}\right.$ is homotopy equivalent to $C_{f}$ and $G^{\prime} \mid X \times\{1\}$ is null-homotopic. By the hypotheses, $G^{\prime} \approx$ const, which implies $G \approx$ const. Since the pair $\left(M(f) \times I, M(f) \times\{0,1\} \cup X^{\prime} \times I\right)$ has the homotopy extension property with respect to any space, we obtain an extension of $G$ over $M(f) \times I$.

Recall that the double mapping cylinder $\operatorname{DM}(f)$ of a map $f: X^{\prime} \rightarrow X$ is the union of two copies of $M(f)$ with two copies of $X^{\prime}$ identified. The natural projection $\operatorname{DM}(f) \rightarrow X$ is denoted by $p$.

Lemma 3. For any map $f: X^{\prime} \rightarrow X$, the mapping cone $C_{p}$ of the natural projection $p: \operatorname{DM}(f) \rightarrow X$ is homeomorphic to the reduced suspension $S\left(C_{f}\right)$ of the mapping cone of $f$. 
Proof. Notice that $\mathrm{DM}(f)$ is homeomorphic to $X^{\prime} \times I \cup M(f) \times\{0,1\} \subset M(f) \times$ $I$, and $M(p)$ is homeomorphic to $M(f) \times I$. Also $C_{p}$ is homeomorphic to $M(f) \times I /\left(X^{\prime} \times I \cup M(f) \times\{0,1\}\right)$. Since $\Sigma\left(C_{f}\right)=\left(M(f) / X^{\prime}\right) \times I /\left(M(f) / X^{\prime}\right) \times$ $\{0,1\}$, Lemma 3 follows.

Lemma 4. Suppose $E$ is a CW complex and $f: X^{\prime} \rightarrow X$ is a closed surjective map of paracompact Hausdorff spaces. Denote by $\mathscr{S}$ the family of all closed subsets $B$ of $X$ such that $q_{f_{A}}^{*}:\left[C_{f_{A}}, E\right] \rightarrow\left[M\left(f_{A}\right), E\right]$ and $f_{A}^{*}:[A, E] \rightarrow$ $\left[f^{-1}(A), E\right]$ are monic for any closed subset $A$ of $B$. If for any closed subset $B$ of $X$ and for any neighborhood $U$ of $B$ there is an open neighborhood $V$ of $B$ in $U$ such that $\operatorname{cl}(V)-V \in \mathscr{S}$, then the image of $f^{*}:[X, E] \rightarrow\left[X^{\prime}, E\right]$ is precisely the set of all homotopy classes $[g]$ such that $g \mid f^{-1}(x) \approx$ const for all $x \in X$.

Proof. It suffices to show that any map $g: X^{\prime} \rightarrow E$ such that $g \mid f^{-1}(x) \approx$ const for all $x \in X$ extends over $M(f)$. Without loss of generality we may assume that $E$ is an ANE for paracompact spaces (see [D-K]). Let $\pi: M(f) \rightarrow X$ be the projection. Fix $x \in X$. Since $g \mid f^{-1}(x) \approx$ const, there exists an extension $g^{\prime}: X^{\prime} \cup \pi^{-1}(x) \rightarrow E$ of $g$. Define $g^{\prime \prime}: X^{\prime} \cup \pi^{-1}(x) \cup X \rightarrow E$ by $g^{\prime \prime} \mid X^{\prime} \cup$ $\pi^{-1}(x)=g^{\prime}$ and $g^{\prime \prime}(X)=g^{\prime}(x) ; g^{\prime \prime}$ extends over a neighborhood $U$ of $X^{\prime} \cup \pi^{-1}(x) \cup X$ in $M(f)$. Choose a neighborhood $V_{x}$ of $x$ in $X$ such that $\pi^{-1}\left(V_{x}\right) \subset U$. Having done that for all $x$ in $X$, we choose a locally finite cover $\left\{A_{s}: s \in S\right\}$ of $X$ consisting of closed sets, which is a refinement of $\left\{V_{x}: x \in X\right\}$. Then, for each $s \in S$, we choose a map $g_{s}: X^{\prime} \cup \pi^{-1}\left(A_{s}\right) \rightarrow E$ such that $g_{s} \mid X^{\prime}=g$ and $g_{s}\left(A_{s}\right)$ is a one-point set.

If $g^{\prime}: X^{\prime} \cup \pi^{-1}(A) \rightarrow E$ is an extension of $g(A$ closed in $X)$ and $s \in$ $S$, then there is an extension $g^{\prime \prime}$ of $g^{\prime}$ over $X^{\prime} \cup \pi^{-1}(U)$ for some closed neighborhood $U$ of $A$. Choose an open neighborhood $V$ of $A$ in $U$ such that $\operatorname{cl}(V)-V \in \mathscr{S}$. By Lemma 2,

$$
g^{\prime \prime}\left|\pi^{-1}\left((\mathrm{cl}(V)-V) \cap A_{s}\right) \approx g_{s}\right| \pi^{-1}\left((\operatorname{cl}(V)-V) \cap A_{s}\right)
$$

rel. $f^{-1}\left((\operatorname{cl}(V)-V) \cap A_{s}\right)$. Since $g_{s} \mid \pi^{-1}\left((\operatorname{cl}(V)-V) \cap A_{s}\right)$ extends over $\pi^{-1}\left(A_{s}\right)$, $g^{\prime \prime}$ extends over $\pi^{-1}\left(A_{s}\right)$. Thus we have an extension $g^{\prime \prime \prime}: X^{\prime} \cup \pi^{-1}\left(A \cup A_{s}\right)$ $\rightarrow E$ of $g$. By well-ordering $S$ and transfinite induction, we can construct an extension $G: M(f) \rightarrow E$ of $g$.

Proof of Theorems A and B. We are going to prove the following statement which implies both Theorems A and B:

(*) Suppose $\left\{E_{k}\right\}_{k \geq 1}$ is a CW spectrum and $f: X^{\prime} \rightarrow X$ is a closed surjective map of paracompact Hausdorff spaces such that, for some integer $n$, $f^{*}: E^{n}(x) \rightarrow E^{n}\left(f^{-1}(x)\right)$ is an isomorphism for all $x \in X$. Denote by $\mathscr{S}$ the family of all closed subsets $B$ of $X$ such that $f^{*}: E^{n-1}(A) \rightarrow E^{n-1}\left(f^{-1}(A)\right)$ is an epimorphism, and $f^{*}: E^{n}(A) \rightarrow E^{n}\left(f^{-1}(A)\right)$ is a monomorphism for all closed subsets $A$ of $B$. If for any closed subset $B$ of $X$ and for any 
neighborhood $U$ of $B$ there is an open neighborhood $V$ of $B$ in $U$ such that $\operatorname{cl}(V)-V \in \mathscr{S}$, then $f^{*}: E^{n}(X) \rightarrow E^{n}\left(X^{\prime}\right)$ is an isomorphism and $f^{*}: E^{n+1}(X) \rightarrow E^{n+1}\left(X^{\prime}\right)$ is a monomorphism.

Without loss of generality, assume $\left\{E_{k}\right\}_{k \geq 1}$ is an $\Omega$-spectrum. Now, $E^{n}(Z)$ $=\left[Z, E_{n}\right]$ for any space $Z$.

Notice that $f^{*}: E^{n}(X) \rightarrow E^{n}\left(X^{\prime}\right)$ is an isomorphism by Lemma 4. Indeed, $f_{A}^{*}:\left[A, E_{n}\right] \rightarrow\left[f^{-1}(A), E_{n}\right]$ is monic for each $A \in \mathscr{S}$, and Lemma 1 implies that $q_{f_{A}}^{*}:\left[C_{f_{A}}, E_{n}\right] \rightarrow\left[M\left(f_{A}\right), E_{n}\right]$ is monic for each $A \in \mathscr{S}$.

So it remains to show that $f^{*}: E^{n+1}(X) \rightarrow E^{n+1}\left(X^{\prime}\right)$ is a monomorphism. Suppose $g, h: X \rightarrow E_{n+1}$ are two maps such that $g f \approx h f$. Then there is a map $H: \operatorname{DM}(f) \rightarrow E_{n+1}$ such that $H$ restricted to one copy of $X$ equals $g$ and $H$ restricted to the other copy of $X$ equals $h$. It suffices to show that $H$ extends over $M(p)$, where $p: \operatorname{DM}(f) \rightarrow X$ is the natural projection. This is easily seen if one notices that $(M(p), \mathrm{DM}(f))$ is homeomorphic to $\left(M(f) \times I, X^{\prime} \times I \cup M(f) \times\{0,1\}\right)$. Then $H|X \times\{0\}=g, H| X \times\{1\}=h$, and any extension of $H$ over $M(f) \times I$ would produce a homotopy from $g$ to $h$ when restricted to $X \times I$.

Notice that $H \mid p^{-1}(x)$ is null-homotopic for all $x \in X$. Indeed, $p^{-1}(x)$ is the suspension $\Sigma f^{-1}(x)$ of $f^{-1}(x)$ and $\left[\Sigma f^{-1}(x), E_{n+1}\right]=\left[f^{-1}(x), \Omega E_{n+1}\right]=$ $\left[f^{-1}(x), E_{n}\right]=\left[\{x\}, E_{n}\right]$. To be able to apply Lemma 4, we need to check that for each $A \in \mathscr{S}$, both $q_{p_{A}}^{*}:\left[C_{p_{A}}, E_{n+1}\right] \rightarrow\left[M\left(p_{A}\right), E_{n+1}\right]$ and $p_{A}^{*}:\left[A, E_{n+1}\right] \rightarrow$ $\left[p^{-1}(A), E_{n+1}\right]$ are monic. The latter is clear, since $p$ is a retraction. By Lemma 3, $\left[C_{p_{A}}, E_{n+1}\right]=\left[S\left(C_{f_{A}}\right), E_{n+1}\right]=\left[C_{f_{A}}, \Omega E_{n+1}\right]=\left[C_{f_{A}}, E_{n}\right]$ (here $C_{p_{A}}$ and $C_{f_{A}}$ are considered as pointed spaces with obvious base points). The proof can be completed by showing that any map $u$ from $C_{f_{A}}$ to $E_{n}$ is null-homotopic (this implies that all pointed maps from $C_{p_{A}}$ to $E_{n+1}$ are null-homotopic, and since all components of $E_{n+1} \cong \Omega E_{n+2}$ are of the same homotopy type, all unpointed maps from $C_{p_{A}}$ to $E_{n+1}$ are null-homotopic). Since $(u \mid A) f \approx$ const, $u \mid A \approx$ const by the first part of the Theorem. Thus $u$ factors (up to homotopy) as $C_{f_{A}} \rightarrow C_{f_{A}} / A=\Sigma f^{-1}(A) \rightarrow E_{n}$. Since $f^{*}: E^{n-1} \cdot(A) \rightarrow E^{n-1}\left(f^{-1}(A)\right)$ is an epimorphism, the map $\Sigma f^{-1}(A) \rightarrow E_{n}$ factors (up to homotopy) as $\Sigma f^{-1}(A) \rightarrow$ $\Sigma(A) \rightarrow E_{n}$. Since $C_{f_{A}} \rightarrow \Sigma(A)$ factors through the cone over $A, u \approx$ const .

Statement $(*)$ obviously implies Theorem B. Theorem A can be proved by induction on $m=$ Ind $X$. If $m=0$, we use Statement $(*)$ with $\mathscr{S}$ being empty and $n=m_{0}$. If Theorem A holds for $m \leq k$ and Ind $X=k+1$, we use Statement $(*)$ with $\mathscr{S}=\{B \in \operatorname{cl}(B) \subset X \mid$ Ind $B \leq k\}$ and $n=k+1+m_{0}$.

The authors are grateful to A. N. Dranišnikov for pointing out an error in the first version of the paper. Also, he noticed that the projection $\pi: S^{1} \rightarrow[-1,1]$ $(\pi(x, y)=x)$ is an example satisfying the hypotheses of Theorem A for $m=$ $m_{0}=1$ (ordinary cohomology is used here) such that $\pi^{*}: H^{m+m_{0}-1}([-1,1]) \rightarrow$ $H^{m+m_{0}-1}\left(S^{1}\right)$ is not an isomorphism. 


\section{REFERENCES}

[B] J. Boardman, Stable homotopy theory, mimeographed notes, Johns Hopkins University, Baltimore, 1970.

[D] A. Dold, Lectures on algebraic topology, Springer-Verlag, Berlin, 1972.

[D-K] J. Dydak and G. Kozlowski, A generalization of the Vietoris-Begle theorem, Proc. Amer. Math. Soc. 102 (1988), 209-212.

[Dr] A. N. Dranišnikov, On a problem of P. S. Aleksandrov, Mat.-Sb. 135 (1988), 551-557.

[D-S] J. Dydak and J. Segal, Shape theory: an introduction, Lecture Notes in Math., vol. 688, Springer-Verlag, Berlin, 1978, pp. 1-150.

[G] B. I. Gray, Homotopy theory, Academic Press, New York, 1975.

[K] J. E. Keesling, A non-movable trivial-shape decomposition of the Hilbert cube, Bull. Acad. Polon. Sci. Ser. Sci. Math. Astronom. Phys. 23 (1975), 997-998.

[Ko] G. Kozlowski, Images of ANR's, mimeographed notes, University of Washington, Seattle, 1974.

[S] E. Spanier, Algebraic topology, McGraw-Hill, New York, 1966.

[Sw] R. M. Switzer, Algebraic topology-homotopy and homology, Springer-Verlag, Berlin, 1975.

[T] J. L. Taylor, A counterexample in shape theory, Bull. Amer. Math. Soc. 81 (1975), 629-632.

[W] G. W. Whitehead, Elements of homotopy theory, Springer-Verlag, Berlin, 1978.

Department of Mathematics, University of Tennessee, Knoxville, Tennessee 37996

Division of Mathematics, Auburn University, Auburn, Alabama 36849 